\title{
A Theory of Wave Devouring Propulsion
}

\author{
(1st Report) \\ - Thrust Generation by a Linear Wells Turbine- -
}

by Hiroshi Isshiki*, Member

\begin{abstract}
Summary
In the present paper, two aspects of wave energy utilization for ship propulsion or so-called "Wave Devouring Propulsion"** are clarified.

(1) From a general viewpoint of conversion of the wave energy into the thrust, the possibility of the wave devouring propulsion is proved by considering the property of the ideal thrust generated by incoming waves and that of the drag required to propel a ship.

(2) As a realization of the wave devouring propulsion, the thrust generation by an oscillating hydrofoil advancing in waves is discussed. This problem has already been discussed by $\mathrm{Wu}^{15), 16}$, but the free surface effect is not included in his theory. When the conversion of the wave power into the propulsive power is aimed at through interaction between a wave and a converter, for example, a hydrofoil, it seems to the author that the free surface effect should not be neglected, since the thrust increase due to the wave power absorption and the wave resistance increase are both proportional to the square of the wave amplitude. In the present paper, Wu's theory is improved by including the free surface effect approximately, and some numerical results are given for a non-oscillating hydorfoil advancing in waves or "A Linear Wells Turbine".
\end{abstract}

\section{Introduction}

The conversion of wave power into propulsive power may be one of the most interesting problems for naval architects. Although the increase of ship speed in following seas is well-known, the ship speed, in general, decreases in head seas. This phenomenon is explained as "Resistance Increase", and is too well-known to naval architects. Hence, the possibility of thrust increase in head seas has long been ignored by naval architects.

* Technical Research Institute, Hitachi Shipbuilding \& Engineering Co., Ltd., Osaka, Japan

** According to Prof. M. Bessho of National Defense Academy of Japan, this terminology was proposed by Prof. T. Y. Wu of California Institute of Technology, when they discussed privately on the subject on the occasion of the 13th ONR Symposium held in Tokyo in 1980.
According to the author's survey, some inventions, observations and investigations related to the wave devouring propulsion have been reported as described in the following.

Descriptions about the first attempt by Herman F. L. Linden on boats propelled by wave motion at the end of the last century are found in refs. 11) and 17). He used flexible fins as shown in Fig. 1, and 13 and $24 \mathrm{ft}$ boats named Autonaut are reported to reach a speed of 3

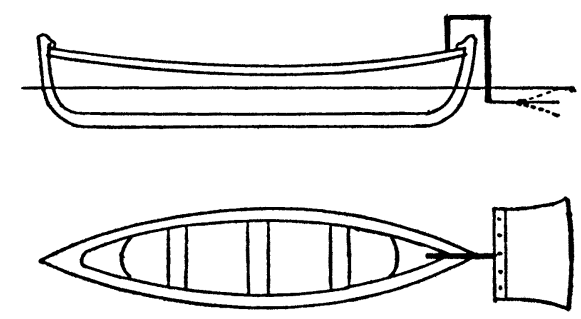

Fig. 1a Drawing from Linden's British patent specification, 1895. (From ref. 17)) 


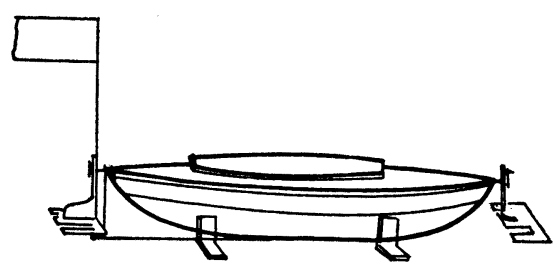

Fig. 1b A drawing of the Autonaut showing the arrangement of fins and steering flag. (From ref. 17))

and 4 knots respectively against wind and sea. But this invention seems to have not been paid much attention by naval architects.

In ref. 7), Longuet-Higgins gives an example of a submerged body like a sand-bar which moves against short and steep waves at a mean speed of $1.2 \mathrm{~cm} / \mathrm{s}$ when wave breaking takes place behind the body. According to his description, this phenomenon is qualitatively similar to "the well-known behavior of offshore sand-bars", and a theory is proposed on the basis of the energy and momentum balance when wave breaking takes place.

In refs. 15) and 16), Wu describes that sea gulls and pelicans are seen to skim ocean waves over a long distance without making noticeable flapping of their wings as in ordinary flight. He also refers to a study on migrating salmon by M. F. M. Osborne. And Wu explores theoretically the possibility to improve propulsive efficiency of a body moving through waves by using oscillating foil propulsors. The theory is based on previous researches by Lighthill' ${ }^{6)}$ and $\mathrm{Wu}^{13) 14)}$.

Huse reports in his paper ${ }^{4}$ that, for certain platforms and sea states, model tests have shown the towing resistance in head seas to be smaller than in calm water, and he explains the phenomenon by introducing a viscous drag force.

Recently, Terao ${ }^{12)}$ succeeded to make a model or "Terao Model" which moved at a fairly high speed against waves without any power supply. His model is made of a plate, called "deck", floating on water surface and two hydrofoils placed under the deck. The details of his model and the experimental results are reported in his paper. The Mechanism of the thrust generation of Terao model has not yet been fully clarified. Its theoretical explanation is one of the motives of the author's present study, though he has not yet succeeded in it.

Terao has also succeeded to make a model similar to the above mentioned model by Herman F. L. Linden.

The author has long been interested in the oscillating foil propulsion of a ship ${ }^{5)}$, since he was ordered to investigate the subject by his superiors of his company about 5 years ago. In the present paper, the author proposes the possibility of the wave devouring propulsion through a general viewpoint of energy conversion. And as a realization of the wave devouring propulsion, the thrust generation by an oscillating hydrofoils in waves is discussed on the basis of Wu's theory ${ }^{15), 16)}$. Wu's theory is developed by introducing the free surface effect on the basis of Maruo's theory ${ }^{9}$ for resistance increase of a ship in waves. Some numerical results are given for the thrust generation by a linear Wells turbine, or a non-oscillating plate foil, advancing in waves.

\section{A discussion of the wave devouring propulsion from a general viewpoint of the wave energy conversion ${ }^{5)}$}

In this section, the possibility of the wave devouring propulsion is proved through a discussion which is based on a general viewpoint of the wave energy conversion and does not specify the type of the energy converter.

For the sake of simplicity, a two-dimensional problem is discussed instead of the actual three dimensional one, and the resistance increase or the wave drifting force is estimated by assuming the advance velocity to be equal to zero.

Furthermore, if the complete absorption of the incoming wave with the amplitude $a$ is assumed, the wave drifting force $\Delta R$ is given as $^{1), 8)}$

$$
\Delta R=\frac{\rho g}{4}|a|^{2} B,
$$

where $\rho, g$ and $B$ are the density of water, acceleration of gravity and breadth of the twodimensional body or ship respectively. On the other hand, the wave power $\bar{P}_{W}^{+}$absorbed in this case is given as

$$
\bar{P}_{W}^{+}=\Delta R c \text {, }
$$

where $c$ is the phase velocity of the wave. Hence, the thrust $\bar{T}_{E}$ obtained by converting the absorbed wave power into the thrust may be written as

$$
\bar{T}_{E}=\eta_{E} \frac{\bar{P}_{W}^{+}}{U}=\eta_{E} \Delta R \frac{c}{U},
$$

where $\eta_{E}$ and $U$ are the efficiency of conversion and the advance velocity of the ship respectively. Therefore, the actual thrust $\bar{T}_{a}$ is obtained by subtracting $\Delta R$ from $\bar{T}_{E}$, that is,

$$
\bar{T}_{a}=\bar{T}_{E}-\Delta R=\Delta R\left(\eta_{E} \frac{c}{U}-1\right) .
$$

It must be noticed that $\bar{T}_{a}$ is proportional to $1 / U$.

From eq. (4), a critical speed $U_{\text {or }}$ may be 
defined by a condition $T_{a}=0$ as

$$
U_{c r}=\eta_{E} c=\eta_{E} \sqrt{\frac{\lambda g}{2 \pi}},
$$

where $\lambda$ is the wave length. According to eq. (5), $U_{c r}$ is proportional to $\sqrt{\lambda}$ and takes pretty large value when $\lambda$ is large. For example, it becomes as large as about $25 \mathrm{kt}$ when $\lambda$ is equal to $100 \mathrm{~m}$. This property of $U_{c r}$ may be important, since $\bar{T}_{a}$ becomes positive for $U<U_{c r}$.

The resistance $R$ of the ship may be written as

$$
R=\frac{\rho}{2} U^{2} B d C_{D}
$$

where $d$ and $C_{D}$ are the draft and the drag coefficient of the ship. Hence, the velocity $U_{s}$ of the self-propulsion speed $U_{s}$ is the solution of the equation:

$$
\bar{T}_{a}=R
$$

or

$$
\Delta R\left(\eta_{E} \cdot \frac{c}{U_{s}}-1\right)=\frac{\rho}{2} U_{s}^{2} B d C_{D} .
$$

Since $\bar{T}_{a}$ is proportional to $1 / U$ and $R$ to $U^{2}$, the solution $U_{s}$ of eq. (7) or eq. (8) dose always exist as shown in Fig. 2.

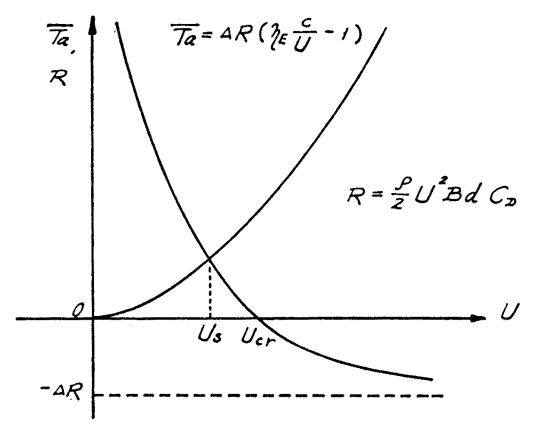

Fig. 2 Self-propulsion velocity $U_{s}$ and critical velocity $U_{c r}$.

In conclusion, the absorbed wave power must be converted into the thrust by some device such as screw propeller, linear propeller ${ }^{6), 13) \sim 16)}$, wave radiation propeller ${ }^{1), 7)}$, etc. And when the thrust, which has, in principle, the property of being inversely proportional to the advance speed, is larger than the wave resistance increase, the positive thrust is obtained from the wave. Furthermore, it may be said that there can exist a velocity at which the ship moves against waves without any power supply.

\section{Thrust generation by an oscillating hy- drofoil in waves}

In this section, the thrust generation by an oscillating two-dimensional hydrofoil in waves is discussed on the basis of Wu's theory ${ }^{13)}{ }^{16)}$, and the two-dimensional version of Maruo's theory for the wave drifting force ${ }^{8), 9)}$ is introduced to include the free surface effect approximately.

3.1 Wu's theory for an oscillating two-dimensional hydrofoil in waves

A two-dimensional hydrofoil of chord $2 l$ and submerged at a mean depth $h_{1}$ moves horizontally with velocity $U$ in a sinusoidal gravity wave of amplitude $a$ and wave length $\lambda$. In terms of the body coordinate system $(x, z)$ as shown in Fig. 3 , the wave profile $\zeta_{0}$, the velocity potential $U x+\phi_{0}(x, z, t)$ and the pressure $p_{0}(x, z, t)$ of the undisturbed wave may be written as

$$
\begin{aligned}
& \zeta_{0}=a \exp \left[i\left(\omega_{0} t\langle\mp\rangle k x\right)\right] \\
& \phi_{0}=\frac{i g a}{\sqrt{g k}} \exp \left[i\left(\omega_{0} t\langle\mp\rangle k x\right)+k z\right] \\
& p_{0}=\rho g a \exp \left[i\left(\omega_{0} t\langle\mp\rangle k x\right)+k z\right]-\rho g z
\end{aligned}
$$

where $i=\sqrt{-1}$

$$
\begin{aligned}
& k=\text { wave number }=2 \pi / \lambda \\
& \omega_{0}=\text { encounter frequency }=\sqrt{g k}\langle \pm\rangle k U(10 \mathrm{~b})
\end{aligned}
$$

and the upper and lower signs in \langle\rangle correspond to the heading and following seas respectively. The wave amplitude $a$ is assumed small such that $k a \ll 1$. The $z$-component of the wave velocity at $z=-h_{1}$ (which coincides with the mean position of the hydrofoil) is given as

$$
\begin{aligned}
v_{0}\left(x,-h_{1}, t\right) & \equiv V_{0}(x, t)=\frac{\partial \phi_{0}}{\partial z} \\
& =i A_{0} \exp \left[i\left(\omega_{0} t\langle\mp\rangle k x\right)\right]
\end{aligned}
$$

where

$$
A_{0}=\sqrt{g k} a \exp \left(-k h_{1}\right) .
$$

As a simplifying assumption, the ratio $\epsilon=A_{0} / U$ of the orbital wave velocity to the mean free stream velocity is taken to be small so that the $x$-component orbital velocity $u_{0}=\partial \phi_{0} / \partial x$ may be neglected in comparison with $U$ in formulating the present linear theory.

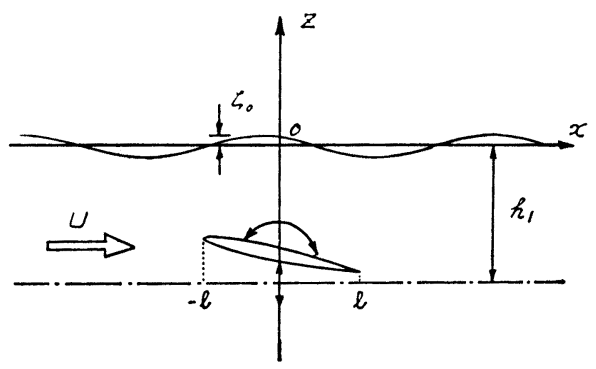

Fig. 3 Body coordinate system for a twodimensional hydrofoil oscillating in a regular wave. 
Although a ship model with a hydrofoil attached to the model with fiexible rods can start moving against oncoming waves according to experiments of Prof. Y. Terao of Tokai University, this phenomenon may not be explained by the present linearlized theory.

In the following, Wu's theory is summarized and supplemented a little. In Wu's theory, the foil-thickness effect is neglected, since the effect is secondary from the viewpoint of potential theory. But, in practical applications, enough attentions to the effect, especially to the shape of the leading edge, should be paid to avoid the flow separation.

The unsteady motion of the hydrofoil assumes the form

$$
z=h(x, t)=\hat{h}(x) \exp (i \omega t) \quad(-l<x<l),
$$

where the circular frequency $\omega$ is arbitrary. With the resultant flow velocity denoted by $\left(U+u_{0}+u_{1}, v_{0}+v_{1}\right)$, the linearlized kinematical boundary condition on the foil may be written as

$$
\begin{aligned}
& \begin{array}{l}
v_{1}(x, \pm 0, t) \equiv V_{1}(x, t)=V(x, t)-V_{0}(x, t) \\
(|x|<l)
\end{array} \\
& V(x, t)=D h(x, t) \\
& D \equiv \partial / \partial t+U \partial / \partial x,
\end{aligned}
$$

where $V_{0}(x, t)$ is given by eq. (11). When the pressure $p$ is taken to be the sum of the undisturbed pressure $p_{0}$ given by eq. (9c) and its perturbation $p_{1} *$, the perturbation pressure $p_{1}$ satisfies the equations of motion

$$
D u_{1}=-\frac{1}{\rho} \frac{\partial p_{1}}{\partial x}, \quad D v_{1}=-\frac{1}{\rho} \frac{\partial p_{1}}{\partial z} .
$$

Eliminating $u_{1}$ and $v_{1}$ from eq. (15) by using the equation of continuity, the governing equation for $p_{1}$ is given as

$$
\left(\frac{\partial^{2}}{\partial x^{2}}+\frac{\partial^{2}}{\partial z^{2}}\right) p_{1}=0 \text { in fluid. }
$$

The pressure component $p_{0}$ gives no hydrodynamic force or moment on the hydrofoil, since it is continuous across the foil of the zero thickness.

The problem to obtain $p_{1}$ is specified by the boundary conditions

$$
\begin{aligned}
& -\frac{1}{\rho} \frac{\partial p_{1}}{\partial z}=D V_{1}(x, t) \quad(|x|<l, z= \pm 0) \\
& p_{1}=0 \quad(|x|>l, z= \pm 0) \\
& p_{1}=0 \quad(x=l, z= \pm 0) \ldots \text { Kutta condition } \\
& p_{1} \rightarrow 0 \quad \text { as }\left|x^{2}+z^{2}\right| \rightarrow \infty
\end{aligned}
$$

and the free surface condition

* The pressure $p_{1}$ is related to Prandtl's acceleration potential $\phi_{1}$ in Wu's paper $^{15}$ ) by $p_{1}=-\rho \phi_{1}$.

$$
\left(D^{2}+g \frac{\partial}{\partial z}+\nu D\right) p_{1}=0,
$$

where $\nu(=+0)$ is Rayleigh's fictitious viscosity.

For the first order approximation, the free surface condition (18) may be neglected, and the solution which satisfies the above mentioned boundary conditions (17) is known (see eqs. (35), (37), (38), (61) and (62) in ref. 13)). In particular, the value of $p_{1}(x, \pm 0, t)$ at the foil is given by

$$
\begin{gathered}
-\frac{1}{\rho} p_{1}(x, \pm 0, t)= \pm \frac{U}{2} a_{0}\left(\frac{l-x}{l+x}\right)^{1 / 2} \\
\pm \frac{1}{\pi} \oint_{-l}^{l}\left(\frac{l^{2}-x^{2}}{l^{2}-\xi^{2}}\right)^{1 / 2} \frac{\psi_{1}(\xi, t)}{\xi-x} d \xi,
\end{gathered}
$$

where

$$
\begin{aligned}
& \psi_{1}(x, t)=-D \int_{-l}^{x} V_{1}(\xi, t) d \xi \\
& a_{0}=\left[b_{1}-\left(b_{0}+b_{1}\right) \Theta(\sigma)\right]-\left[b_{1}{ }^{\prime}-\left(b_{0}{ }^{\prime}+b_{1}{ }^{\prime}\right) \Theta\left(\sigma_{0}\right)\right] \\
& b_{n}=\frac{2}{\pi} \int_{0}^{\pi} V(l \cos \theta, x) \cos n \theta d \theta \\
& (x=l \cos \theta, n=0,1,2, \cdots) \\
& b_{n}{ }^{\prime}=\frac{2}{\pi} \int_{0}^{\pi} V_{0}(l \cos \theta, x) \cos n \theta d \theta \\
& =\frac{2}{\pi} \int_{0}^{\pi} i A_{0} \exp \left[i\left(\omega_{0} t\langle\mp\rangle k l \cos \theta\right)\right] \cos n \theta d \theta \\
& =\langle \pm\rangle 2 A_{0}(\langle\mp\rangle i)^{n-1} J_{n}(k l) \exp \left(i \omega_{0} t\right) \\
& \Theta(\sigma)=\frac{K_{1}(j \sigma)}{K_{0}(j \sigma)+K_{1}(j \sigma)}=\frac{H_{1}^{(2)}(\sigma)}{H_{1}^{(2)}(\sigma)+i H_{0}^{(2)}(\sigma)} \\
& =\mathscr{I}(\sigma)+i \mathscr{G}(\sigma) \\
& \sigma_{0}=\omega_{0} l / U \\
& \sigma=\omega l / U \text {. }
\end{aligned}
$$

Here, the integral in eq. (19) assumes its Cauchy principal value; $J n(k l)$ is the Bessel function of the first kind; $\Theta(\sigma)$ is the Theodorsen function ${ }^{10)}$, $\mathscr{F}$ and $\mathcal{G}$ being its real and imaginary parts; $K_{n}(\sigma)$ is the modified Bessel function of the second kind; $H_{n}{ }^{(2)}(\sigma)$ is the Hankel function of the second kind; $\sigma$ and $\sigma_{0}$ are the reduced frequencies of the foil and wave motions respectively. There exists a relation between $\sigma_{0}$ and $k l$ as

$$
\sigma_{0}=(\delta k l)^{1 / 2}\langle \pm\rangle k l, \quad \delta=g l / U^{2},
$$

$(1 / \delta)^{1 / 2}$ being the Froude number.

The differential lift distribution $\mathcal{L}$ along the chord is clearly

$$
\begin{aligned}
\mathcal{L}(x, t) & =p(x,-0, t)-p(x,+0, t) \\
& =-2 p_{1}(x,+0, t) \quad(|x|<l) .
\end{aligned}
$$

The integral representation of the lift $L(t)$ and the moment $M(t)$ (about the mid-chord; positive in the nose up sense) are

$$
L(t)=\int_{-l}^{l} \mathcal{L}(x, t) d x
$$




$$
M(t)=-\int_{-l}^{l} \mathcal{L}(x, t) x d x .
$$

When eqs. (19) and (22) are substituted into eqs. (23) and (24), the lift $L(t)$ and the moment $M(t)$ can be written as

$$
\begin{aligned}
L(t)= & \pi \rho U l\left\{-\left[\left(b_{0}+b_{1}\right) \Theta(\sigma)+\frac{i \sigma}{2}\left(b_{0}-b_{2}\right)\right]\right. \\
& \left.+\left[\left(b_{0}{ }^{\prime}+b_{1}{ }^{\prime}\right) \Theta\left(\sigma_{0}\right)+\frac{i \sigma_{0}}{2}\left(b_{0}{ }^{\prime}-b_{2}{ }^{\prime}\right)\right]\right\} \\
M(t)= & \frac{\pi \rho U l^{2}}{2}\left\{\left[b_{1}-\left(b_{0}+b_{1}\right) \Theta(\sigma)+b_{2}\right]\right. \\
& +\frac{i \sigma}{4}\left(b_{1}-b_{3}\right)-\left[b_{1}{ }^{\prime}-\left(b_{0}{ }^{\prime}+b_{1}{ }^{\prime}\right)\right. \\
& \left.\left.\times \Theta\left(\sigma_{0}\right)+b_{2}{ }^{\prime}\right]-\frac{i \sigma_{0}}{4}\left(b_{1}{ }^{\prime}-b_{3}{ }^{\prime}\right)\right\} .
\end{aligned}
$$

In order to obtain eqs. (25) and (26), eqs. (40) and (41) in ref. 13) are used respectively. The substitutions of eqs. (20d) into eqs. (25) and (26) gives

$$
\begin{aligned}
& \frac{L(t)}{\pi \rho U^{2} l}=-\frac{1}{U}\left[\left(b_{0}+b_{1}\right) \Theta(\sigma)+\frac{i \sigma}{2}\left(b_{0}-b_{2}\right)\right] \\
&-2 \frac{A_{0}}{U}\left[W_{1}\left(\sigma_{0}\right)-i W_{2}\left(\sigma_{0}\right)\right. \\
&\left.+\left(\langle\mp\rangle 1+\frac{\sigma_{0}}{k l}\right) J_{1}(k l)\right] \exp \left(i \omega_{0} t\right) \\
& \frac{M(t)}{(1 / 2) \pi \rho U^{2} l^{2}}=-\frac{1}{U}\left[\left(b_{0}+b_{1}\right) \Theta(\sigma)-b_{1}-b_{2}\right. \\
&\left.-\frac{i \sigma}{4}\left(b_{1}-b_{3}\right)\right]-2 \frac{A_{0}}{U}\left[W_{1}\left(\sigma_{0}\right)\right. \\
&-i W_{2}\left(\sigma_{0}\right)-i\left(1\langle\mp\rangle \frac{\sigma_{0}}{k l}\right) \\
&\left.\times J_{2}(k l)\right] \exp \left(i \omega_{0} t\right),
\end{aligned}
$$

where

$$
\begin{aligned}
& W_{1}(\sigma)=[\langle \pm\rangle 1\langle\mp\rangle \mathscr{I}(\sigma)] J_{1}(k l)+\mathcal{G}(\sigma) J_{0}(k l) \\
& W_{2}(\sigma)=\mathscr{F}(\sigma) J_{0}(k l)\langle \pm\rangle \mathcal{G}(\sigma) J_{1}(k l) .
\end{aligned}
$$

The formulas for calculating the thrust $T$, the power required for maintaining the motion $P$ and the kinectic energy imparted to the fluid $E$ may be given as

$$
\begin{aligned}
& T=\int_{-l}^{l} \mathcal{L}(x, t) \frac{\partial h}{\partial x} d x+S \\
& P=-\int_{-l}^{l} \mathcal{L}(x, t) \frac{\partial h}{\partial t} d x \\
& E=-\int_{-l}^{l} \mathcal{L}(x, t) \text { Dhd } x-S U,
\end{aligned}
$$

where $S$ is the leading edge suction and is given as (see eq. (43) in ref. 13))

$$
S=\frac{1}{2} \pi \rho\left(\operatorname{Re}\left[a_{0}\right]\right)^{2} \text {. }
$$

* Eq. (18) in ref. 16) seems to be incorrect. The coefficient $1 / 8$ should be $1 / 2$ as given in eq. (21) in ref. 15).
From the above expressions of $T, P$ and $E$, it is easily shown that there exists the energy relation, that is,

$$
P=T U+E \text {. }
$$

In the following, discussions are limited to the heaving and pitching oscillations of the flatplate hydrofoil. The motion of the foil may be described as

$$
h(x, t)=\left[\frac{1}{2}\left(\xi_{1}+i \xi_{2}\right)+\left(\xi_{3}+i \xi_{4}\right) x\right] \exp (i \omega t),
$$

where $\xi_{1} \ldots, \xi_{4}$ are real coefficients. $\left(\xi_{1}, \xi_{2}\right)$ and $\left(\xi_{3}, \xi_{4}\right)$ represents a heaving and a pitching-aboutmid-chord with their amplitudes

$$
\xi_{h}=\left(\xi_{1}^{2}+\xi_{2}^{2}\right)^{1 / 2}, \quad \xi_{p}=\left(\xi_{3}^{2}+\xi_{4}^{2}\right)^{1 / 2}
$$

and their phase angles

$$
\alpha_{h}=\tan ^{-1} \xi_{2} / \xi_{1}, \quad \alpha_{p}=\tan ^{-1} \xi_{4} / \xi_{3}
$$

respectively. When the expression (35) is substituted into $(14 \mathrm{~b}), V(x, t)$ is now given as

$$
\begin{aligned}
V(x, t)= & {\left[\frac{i(\omega)}{2}\left(\xi_{1}+i \xi_{2}\right)+U\left(\xi_{3}+i \xi_{4}\right)\right] \exp (i \omega t) } \\
& +\left[i \omega\left(\xi_{3}+i \xi_{4}\right) x\right] \exp (i \omega t) .
\end{aligned}
$$

The substitution of eq. (37) into eq. (20c) gives

$$
\begin{aligned}
& b_{0}=U\left[i \sigma\left(\xi_{1}+i \xi_{2}\right)+2\left(\xi_{3}+i \xi_{4}\right)\right] \exp (i \omega t) \\
& b_{1}=U i \sigma\left(\xi_{3}+i \xi_{4}\right) \exp (i \omega t) \\
& b_{n}=0 \text { for } n \geq 2 .
\end{aligned}
$$

The simple geometry of the flat plate foil leads to simplifications in the calculation for thrust and power. In fact, the substitution of eq. (25) into eqs. (30) and (31) yields

$$
\begin{aligned}
T= & \operatorname{Re}[L] \cdot \operatorname{Re}[\partial h / \partial x]+S \\
P= & -\operatorname{Re}\left[\frac{1}{2} i \omega\left(\xi_{1}+i \xi_{2}\right) \exp (i \omega t)\right] \operatorname{Re}[L] \\
& +\operatorname{Re}\left[i \omega\left(\xi_{3}+i \xi_{4}\right) \exp (i \omega t)\right] \operatorname{Re}[M] .
\end{aligned}
$$

Furthermore, if $\omega$ is assumed to be equal to $\omega_{0}$, the mean value of thrust $\vec{T}$, leading edge suction $\bar{S}$, power $\bar{P}$ and energy loss $\bar{E}$ are obtained as

$$
\begin{aligned}
\bar{T}= & \frac{1}{2} \operatorname{Re}\left[L \cdot\left(\frac{\partial h}{\partial x}\right)^{*}\right]+\bar{S} \\
\bar{S}= & \frac{1}{4} \pi \rho\left|a_{0}\right|^{2} \\
\bar{P}= & -\frac{1}{4} \operatorname{Re}\left[i \omega_{0}\left(\xi_{1}+i \xi_{2}\right) \exp \left(i \omega_{0} t\right) L^{*}\right] \\
& +\frac{1}{2} \operatorname{Re}\left[i \omega_{0}\left(\xi_{3}+i \xi_{4}\right) \exp \left(i \omega_{0} t\right) M^{*}\right] \\
\bar{E}= & \bar{P}-\bar{T} U,
\end{aligned}
$$

where the asterisk means to take the complex conjugate of the corresponding quantities.

3.2 Wave drifting force acting on an oscillating hydrofoil in waves

In the following, the two-dimensional version 
of Maruo's theory for the wave drifting force ${ }^{3), 8), 9 \text { ) }}$ is applied to estimate approximately the wave drifting force or resistance increase in head seas and "thrust increase" in following seas.

For simplicity, the wave drifting force is estimated by using the amplitudes of the radiated waves which is generated by the concentrated singularities expressing the lift $L$ and the moment $M$, whose values are calculated without including the free surface effect, that is, according to eqs. (27) and (28).

In the first place, the hydrodynamic pressure $P_{L} \exp \left(i \omega_{0} t\right)$ and $P_{M} \exp \left(i \omega_{0} t\right)$ of the singularities at $\left(x_{1}, z_{1}\right)$ expressing the lift $L=L_{0} \exp \left(i \omega_{0} t\right)$ and the moment $M=M_{0} \exp \left(i \omega_{0} t\right)$ are summarized. $P_{L}$ and $P_{M}$ are obtained as

$$
\begin{aligned}
& P_{L}=L_{0} \frac{\partial P_{F}}{\partial z_{1}} \\
& P_{M}=-M_{0} \frac{\partial\left[P_{L}\right]_{L_{0}=1}}{\partial x_{1}},
\end{aligned}
$$

where $P_{F}$ is the fundamental singularity ${ }^{2)}$ defined by

$$
\begin{aligned}
& \left(\frac{\partial^{2}}{\partial x^{2}}+\frac{\partial^{2}}{\partial z^{2}}\right) P_{F}=\delta\left(x-x_{1}\right) \delta\left(z-z_{1}\right) \\
& \text { in fluid } \quad(47 \mathrm{a}) \\
& {\left[\left(i \omega_{0}+U \frac{\partial}{\partial x}\right)^{2}+g \frac{\partial}{\partial z}+\nu\left(i \omega_{0}+U \frac{\partial}{\partial x}\right)\right] P_{F}=0} \\
& \text { at } z=0
\end{aligned}
$$

since the hydrodynamic pressure $p(x, z, t)$ generated by the source distribution $m(x, z, t)$ and the external force distribution $\left(f_{x}(x, z, t), f_{z}(x, z, t)\right)$ is the solution of the following problem

$$
\begin{aligned}
\left(\frac{\partial^{2}}{\partial x^{2}}+\frac{\partial^{2}}{\partial z^{2}}\right) p= & -\rho\left(\frac{\partial}{\partial t}+U \frac{\partial}{\partial x}+\nu\right) m \\
& +\left(\frac{\partial f_{x}}{\partial x}+\frac{\partial f_{z}}{\partial z}\right) \\
& \text { in fluid } \\
{\left[\left(\frac{\partial}{\partial t}+U \frac{\partial}{\partial x}\right)^{2}+g \frac{\partial}{\partial z}+\nu\left(\frac{\partial}{\partial t}+U \frac{\partial}{\partial x}\right)\right] p=0 } & \text { at } z=0
\end{aligned}
$$

By solving the problem defined by eq. (47), the fundamental singularity $P_{\boldsymbol{F}}$ is obtained as

$$
\begin{aligned}
P_{F}= & \frac{1}{2 \pi}\left\{\ln \sqrt{\left(x-x_{1}\right)^{2}+\left(z-z_{1}\right)^{2}}\right. \\
& \left.-\ln \sqrt{\left(x-x_{1}\right)^{2}+\left(z+z_{1}\right)^{2}}\right\} \\
& -\frac{g}{2 \pi} \int_{0}^{\infty} \frac{\exp \left[i k\left(x-x_{1}\right)+k\left(z+z_{1}\right)\right]}{\left\{-\left(\omega_{0}+k U\right)^{2}+g k+i \nu\left(\omega_{0}+k U\right)\right\}} d k \\
& -\frac{g}{2 \pi} \int_{0}^{\infty} \frac{\exp \left[-i k\left(x-x_{1}\right)+k\left(z+z_{1}\right)\right]}{\left\{-\left(\omega_{0}-k U\right)^{2}+g k+i \nu\left(\omega_{0}-k U\right)\right\}} d k,
\end{aligned}
$$

and $P_{F}$ has the following asymptotic forms

$$
\begin{aligned}
& P_{F} \sim E\left(\frac{1}{4}-\Omega\right) \frac{i}{\sqrt{1-4 \Omega}} \exp \left[i k_{2}-\left(x-x_{1}\right)\right. \\
& \left.+k_{2}-\left(z+z_{1}\right)\right] \quad \text { when } x \rightarrow-\infty \quad \text { (50a) } \\
& P_{F} \sim E\left(\frac{1}{4}-\Omega\right) \frac{i}{\sqrt{1-4 \Omega}} \exp \left[i k_{1}-\left(x-x_{1}\right)\right. \\
& \left.+k_{1}-\left(z+z_{1}\right)\right]-\frac{i}{\sqrt{1+4 \Omega}} \exp \left[-i k_{1}{ }^{+}\left(x-x_{1}\right)\right. \\
& \left.+k_{1}{ }^{+}\left(z+z_{1}\right)\right]+\frac{i}{\sqrt{1+4 \Omega}} \exp \left[-i k_{2}{ }^{+}\left(x-x_{1}\right)\right. \\
& \left.+k_{2}{ }^{+}\left(z+z_{1}\right)\right] \quad \text { when } x \rightarrow \infty, \quad(50 \mathrm{~b})
\end{aligned}
$$

where

$$
\begin{aligned}
& E(x)=\left\{\begin{array}{lll}
0 & \text { when } & x<0 \\
1 & \text { when } & x>0
\end{array}\right\} \\
& \left.k_{1}^{+}\right\}=\frac{\kappa_{0}}{2}(1+2 \Omega \pm \sqrt{1+4 \Omega}) \\
& k_{2}{ }^{-} \\
& \left.k_{1}^{-}\right\}=\frac{\kappa_{0}}{2}(1-2 \Omega \pm \sqrt{1-4 \Omega}) \\
& \left.k_{2}^{-}\right\} \\
& \kappa_{0}=g / U^{2} \\
& \Omega=U \omega_{0} / g .
\end{aligned}
$$

The asymptotic forms of $P_{L}$ and $P_{M}$ may easily be derived from eqs. (45), (46), and (50).

Since the velocity potential $U x+\Phi(x, z) \exp$ $\left(i \omega_{0} t\right)$ is obtained by solving the Bernoulli equation

$$
p=-\rho\left(\frac{\partial}{\partial t}+U \frac{\partial}{\partial x}\right) \Phi \exp \left(i \omega_{0} t\right),
$$

where $p(x, z, t)$ is the hydrodynamic pressure, the wave terms in the asymptotic forms of the velocity potential $U x+\Phi \exp \left(i \omega_{0} t\right)$ which expresses the uniform stream, the incoming wave and the lift and moment singularities at $\left(x_{1}, z_{1}\right)$ may be obtained as

$$
\begin{aligned}
& \Phi \sim \frac{i g a}{\omega_{0}\langle\mp\rangle k U} \exp [\langle\mp\rangle i k x+k z] \\
& -E\left(\frac{1}{4}-\Omega\right) \frac{\left(L_{0}+i M_{0} k_{2}^{-}\right) k_{2}^{-}}{\rho\left(\omega_{0}+U k_{2}^{-}\right) \sqrt{1-4 \Omega}} \\
& \times \exp \left[i k_{2}-\left(x-x_{1}\right)+k_{2}-\left(z+z_{1}\right)\right] \\
& \text { when } x \rightarrow-\infty \\
& \Phi \sim \frac{i g a}{\omega_{0}\langle\mp\rangle k U} \exp [\langle\mp\rangle i k x+k z] \\
& -E\left(\frac{1}{4}-\Omega\right) \frac{\left(L_{0}+i M_{0} k_{1}^{-}\right) k_{1}^{-}}{\rho\left(\omega_{0}+k_{1}-U\right) \sqrt{1-4 \Omega}} \\
& \times \exp \left[i k_{1}-\left(x-x_{1}\right)+k_{1}-\left(z+z_{1}\right)\right] \\
& +\frac{\left(L_{0}-i M_{0} k_{1}{ }^{+}\right) k_{1}{ }^{+}}{\rho\left(\omega_{0}-k_{1}+U\right) \sqrt{1+4 \Omega}} \\
& \times \exp \left[-i k_{1}{ }^{+}\left(x-x_{1}\right)+k_{1}{ }^{+}\left(z+z_{1}\right)\right] \\
& -\frac{\left(L_{0}-i M_{0} k_{2}{ }^{+}\right) k_{2}{ }^{+}}{\rho\left(\omega_{0}-k_{2}{ }^{+} U\right) \sqrt{1+4 \Omega}} \\
& \times \exp \left[-i k_{2}{ }^{+}\left(x-x_{1}\right)+k_{2}{ }^{+}\left(z+z_{1}\right)\right]
\end{aligned}
$$$$
\text { when } x \rightarrow \infty \text {. }
$$

Subsequently, the two-dimensional version 
of Maruo's formula for the wave drifting force, that is eq. (5.74) in ref. 9), is derived. In the first place, the two-dimensional form of the oscillatory part $\Phi_{1}(x, y, z) \exp \left(i \omega_{0} t\right)$ of the velocity potential:

$$
\begin{aligned}
\Phi_{1}= & -2 i\left[\int_{-\pi / 2}^{\theta-\pi / 2}-\int_{\pi / 2}^{\theta+\pi / 2}\right] H_{1}\left(\kappa_{1}, \alpha\right) \\
& \times \frac{\kappa_{1} \exp \left[\kappa_{1} z-i \kappa_{1} R \cos (\alpha-\theta)\right]}{\sqrt{1-4 \Omega \cos \alpha}} d \alpha \\
& -2 i \int_{\theta-\pi / 2}^{\theta+\pi / 2} H_{1}\left(\kappa_{2}, \alpha\right) \\
& \times \frac{\kappa_{2} \exp \left[\kappa_{2} z-i \kappa_{2} R \cos (\alpha-\theta)\right]}{\sqrt{1-4 \Omega \cos \alpha}} d \alpha \\
& +0\left(R^{-1}\right) \quad \text { for large } R
\end{aligned}
$$

is obtained (see eq. (5.63) in ref. 9)). In the above expression,

$$
\begin{aligned}
& R=\sqrt{x^{2}+y^{2}}, \quad \theta=\tan ^{-1}(y / x)+\pi \\
& H_{1}(\kappa, \alpha)= \iint_{S} \sigma_{1}\left(x^{\prime}, y^{\prime}, z^{\prime}\right) \\
& \times \exp \left(\kappa z^{\prime}-i \kappa x^{\prime} \cos \alpha-i \kappa y^{\prime} \sin \alpha\right) \\
& \times d S\left(x^{\prime}, y^{\prime}, z^{\prime}\right)
\end{aligned}
$$

$\sigma_{1}=$ strength of the source distributed on the ship surface

$$
\left.\begin{array}{l}
\kappa_{1} \\
\kappa_{2}
\end{array}\right\}=\kappa_{0} \frac{1-2 \Omega \cos \alpha \pm \sqrt{1-4 \Omega \cos \alpha}}{2 \cos ^{2} \alpha} .
$$

The coordinate system is adjusted to coincide with the one adapted in the present paper: the variables $x$ and $y$ are replaced by $-x$ and $-y$ respectively; the uniform stream of the velocity $U$ flows from $x=-\infty$ to $x=+\infty$; the angles $\alpha$ and $\theta$ are measured in counter-clockwise sense from the negative $x$-axis.

In the two-dimensional case, $\sigma_{1}$ is independent of $y^{\prime}$, that is,

$$
\sigma_{1}=\sigma_{1}\left(x^{\prime}, z^{\prime}\right) \text {. }
$$

Substituting eq. (56) into eq. (55b), $H_{1}(\kappa, \alpha)$ can now be written as

$$
\begin{aligned}
H_{1}(\kappa, \alpha)= & \int_{S} \sigma_{1}\left(x^{\prime}, z^{\prime}\right) \\
& \times \exp \left(\kappa z^{\prime}-i \kappa x^{\prime} \cos \alpha\right) \\
& \times d S\left(x^{\prime}, z^{\prime}\right) \delta(\kappa \sin \alpha) \\
= & H_{1}{ }^{\prime}(\kappa, \alpha) \delta(\kappa \sin \alpha),
\end{aligned}
$$

where $\delta$ is Dirac's delta function and the following expression of the delta function is used

$$
\delta(\kappa)=\frac{1}{2 \pi} \int_{-\infty}^{\infty} e^{-i \kappa x} d x .
$$

Substituting eq. (57) into eq. (54) and putting $\theta=0$, the asymptotic expression of the twodimensional velocity potential $\Phi_{1}$ when $x \rightarrow-\infty$ is obtained as

$$
\begin{aligned}
\Phi_{1} \sim & -2 i \int_{-\pi / 2}^{\pi / 2} H_{1}{ }^{\prime}\left(\kappa_{2}, \alpha\right) \delta\left(\kappa_{2} \sin \alpha\right) \\
& \times \frac{\kappa_{2} \exp \left[\kappa_{2} z+i \kappa_{2} x \cos \alpha\right]}{\sqrt{1-4 \Omega \cos \alpha}} d \alpha \\
= & -2 i \int_{-\kappa_{2}(0) \Delta_{\alpha}}^{\kappa_{2}(0) \Delta_{\alpha}} H_{1}{ }^{\prime}\left(\kappa_{2}, \alpha\right) \delta(t) \\
& \times \frac{\kappa_{2} \exp \left[\kappa_{2} z+i \kappa_{2} x \cos \alpha\right]}{\sqrt{1-4 \Omega \cos \alpha}} \\
& \times \frac{d t}{\kappa_{2}{ }^{\prime}(\alpha) \sin \alpha+\kappa_{2}(\alpha) \cos \alpha} \\
= & -2 i H_{1}{ }^{\prime}\left(\kappa_{2}(0), 0\right) \frac{\exp \left[\kappa_{2}(0) z+i \kappa_{2}(0) x\right]}{\sqrt{1-4 \Omega}} .
\end{aligned}
$$

Similarly, the asymptotic expression when $x \rightarrow$ $+\infty$ is obtained as

$$
\begin{aligned}
\Phi_{1} \sim & -2 i H_{1}{ }^{\prime}\left(\kappa_{1}(0), 0\right) \frac{\exp \left[\kappa_{1}(0) z+i \kappa_{1}(0) x\right]}{\sqrt{1-4 \Omega}} \\
& +2 i H_{1}{ }^{\prime}\left(\kappa_{1}(\pi), \pi\right) \frac{\exp \left[\kappa_{1}(\pi) z-i \kappa_{1}(\pi) x\right]}{\sqrt{1+4 \Omega}} \\
& -2 i H_{1}{ }^{\prime}\left(\kappa_{2}(\pi), \pi\right) \frac{\exp \left[\kappa_{2}(\pi) z-i \kappa_{2}(\pi) x\right]}{\sqrt{1+4 \Omega}} .
\end{aligned}
$$

From eqs. (51b), (51c) and (55d), $\kappa_{j}(\alpha)(j=1,2$; $\alpha=0, \pi)$ and $k_{j}^{ \pm}(j=1,2)$ are related as

$$
\left.\begin{array}{l}
\kappa_{j}(0)=k_{j}^{-} \\
\kappa_{j}(\pi)=k_{j}^{+}
\end{array}\right\} \quad \text { for } j=1,2 .
$$

Now, the two-dimensional form of Maruo's formula for the resistance increase $\Delta R$ in waves (see eq. (5.74) in ref. 9):

$$
\begin{aligned}
\Delta R= & 2 \pi \rho\left[\int_{-\pi / 2}^{-\alpha_{0}}+\int_{\alpha_{0}}^{\pi / 2}-\int_{\pi / 2}^{3 \pi / 2}\right] \\
& \times\left|H_{1}\left(\kappa_{1}, \alpha\right)\right|^{2} \frac{\kappa_{1}^{2} \cos \alpha}{\sqrt{1-4 \Omega \cos \alpha}} d \alpha \\
& +2 \pi \rho \int_{\alpha_{0}}^{2 \pi-\alpha_{0}}\left|H_{1}\left(\kappa_{2}, \alpha\right)\right|^{2} \frac{\kappa_{2}^{2} \cos \alpha}{\sqrt{1-4 \Omega \cos \alpha}} d \alpha \\
& -i \pi \rho c r k\left[H_{1}\left(k, \lambda^{\prime}\right)-H_{1} *\left(k, \chi^{\prime}\right)\right] \cos \chi
\end{aligned}
$$

is derived as follows. In the expression (61), $\alpha_{0}$ is

$$
\alpha_{0}=\left\{\begin{array}{ll}
0 & \text { for } \Omega \leq 1 / 4 \\
\cos ^{-1}(1 / 4 \Omega) & \text { for } \Omega>1 / 4,
\end{array}\right\}
$$

and $\chi$ is the direction of the incident wave $\Phi_{0}$ :

$$
\Phi_{0}=c r \exp [k z+i k(x \cos x+y \sin \chi)] .
$$

Substitution of eq. (57) into eq. (61) then leads the form of $\Delta R$ for an infinite cylinder moving in a heading $(\chi=\pi)$ or following $(\chi=0)$ seas

$$
\Delta R=2 \pi \rho\left[E(1-4 \Omega)\left|H_{1}{ }^{\prime}\left(\kappa_{1}(0), 0\right)\right|^{2}\right.
$$




$$
\begin{aligned}
& \times \frac{\kappa_{1}(0)}{\sqrt{1-4 \Omega}}+\left|H_{1}{ }^{\prime}\left(\kappa_{1}(\pi), \pi\right)\right|^{2} \frac{\kappa_{1}(\pi)}{\sqrt{1+4 \Omega}} \\
& +E(1-4 \Omega)\left|H_{1}{ }^{\prime}\left(\kappa_{2}(0), 0\right)\right|^{2} \frac{\kappa_{2}(0)}{\sqrt{1-4 \Omega}} \\
& -\left|H_{1}{ }^{\prime}\left(\kappa_{2}(\pi), \pi\right)\right|^{2} \frac{\kappa_{2}(\pi)}{\sqrt{1+4 \Omega}}+\frac{1}{2} i c r k \\
& \left.\times\left\{H_{1}{ }^{\prime}(k, \gamma)-H_{1}{ }^{\prime} *(k, \gamma)\right\} \cos \gamma\right] \delta(0) .
\end{aligned}
$$

Comparing eq. (64) with eq. (17) in ref. 8) for zero advance velocity, $2 \pi \delta(0)$ in eq. (64) is replaced by 1 , and the two-dimensional value $\Delta R_{2}$ corresponding to $\Delta R$ is obtained as

$$
\begin{aligned}
\Delta R_{2}= & \rho\left[E(1-4 \Omega)\left|H_{1}{ }^{\prime}\left(\kappa_{1}(0), 0\right)\right|^{2}\right. \\
& \times \frac{\kappa_{1}(0)}{\sqrt{1-4 \Omega}}+\left|H_{1}{ }^{\prime}\left(\kappa_{1}(\pi), \pi\right)\right|^{2} \frac{\kappa_{1}(\pi)}{\sqrt{1+4 \Omega}} \\
& +E(1-4 \Omega)\left|H_{1}{ }^{\prime}\left(\kappa_{2}(0), 0\right)\right|^{2} \frac{\kappa_{2}(0)}{\sqrt{1-4 Q}} \\
& -\left|H_{1}{ }^{\prime}\left(\kappa_{2}(\pi), \pi\right)\right|^{2} \frac{\kappa_{2}(\pi)}{\sqrt{1+4 \Omega}}-\frac{1}{2} i c \gamma k \\
& \left.\times\left\{H_{1}{ }^{\prime}(k, \gamma)-H_{1}{ }^{*}(k, \gamma)\right\} \cos \gamma\right] .
\end{aligned}
$$

From the comparison of eq. (53) with eqs. (59) and (63), $\mathrm{cr}^{\prime}, H_{1}{ }^{\prime}\left(\kappa_{j}(0), 0\right)$ and $H_{1}{ }^{\prime}\left(\kappa_{j}(\pi), \pi\right)$ $(j=1,2)$ is calculated by

$$
\begin{aligned}
c r=\frac{g a}{\left|\omega_{0}\langle\mp\rangle k U\right|} & \\
H_{1}{ }^{\prime}\left(\kappa_{j}(0), 0\right)= & \frac{-i\left(L_{0}+i M_{0} k_{j}-\right) k_{j}-}{2 \rho\left(\omega_{0}+U k_{j}{ }^{-}\right)} \exp \left[-k_{j}-h\right. \\
& \left.-i\left(\frac{\pi}{2}-\arg \left(\omega_{0}\langle\mp\rangle k U\right)\right)\right] \quad(66 \mathrm{~b} \\
H_{1}{ }^{\prime}\left(\kappa_{j}(\pi), \pi\right)= & \frac{-i\left(L_{0}-i M_{0} k_{j}{ }^{+}\right) k_{j}{ }^{+}}{2 \rho\left(\omega_{0}-k_{j}{ }^{+} U\right)} \exp \left[-k_{j}{ }^{+} h_{1}\right. \\
& \left.-i\left(\frac{\pi}{2}-\arg \left(\omega_{0}\langle\mp\rangle k U\right)\right)\right] . \quad(66 \mathrm{c})
\end{aligned}
$$

\section{Some numerical results for a linear Wells turbine}

In order to clarify the effect of oscillations of a hydrofoil, the details of the oscillations must be specified by solving any optimum problems as pointed by $\mathrm{Wu}^{15), 16)}$. A solution of an optimum problem has already been given by $\mathrm{Wu}$, but the author wants to treat problems of optimization in future.

In the present paper, some numerical calculations are conducted for a non-oscillating foil advancing in waves or "linear Wells turbine". The mechanism of thrust generation by a linear Wells turbine is illustrated in Fig. 4.

In Fig. 5, the performance results for a linear Wells turbine of chord $2 l=0.328 \mathrm{~m}$ advancing in head seas with a velocity of $U=1 \mathrm{~m} / \mathrm{s}$ are shown.

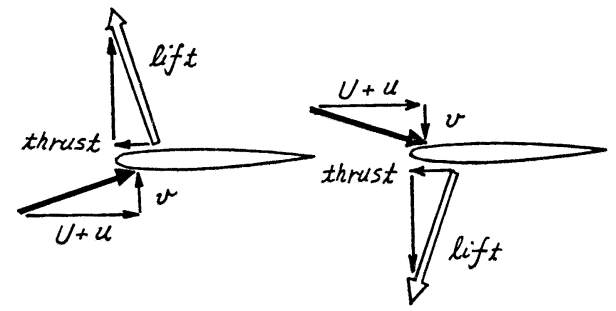

Fig. 4 Thrust generation by a linear Wells turbine.

In Figs. 5 9, $\bar{T}_{a}, \bar{P}_{W}^{ \pm}$and $\eta_{W}^{ \pm}$are as follows

$\bar{T}_{a}=$ actual mean thrust

$$
=\bar{T}-\Delta R_{2}
$$

$\bar{P}_{\mathrm{W}}^{ \pm}=$wave power

$$
=\frac{\rho g}{2}|a|^{2}\left(c_{g} \pm U\right)
$$

$\eta_{W D}^{ \pm}=$wave devouring efficiency

$$
=\bar{T}_{a} U / \bar{P}_{\mathrm{W}}^{+}
$$

where the upper and lower signs in \pm refer to heading and following seas respectively, and

$c_{g}=$ group velocity of the wave

$$
=\frac{1}{2} \sqrt{\frac{\lambda g}{2 \pi}}
$$

In Fig. 5, when the wave length $\lambda$ tends to $15+0 \mathrm{~m}$, the resistance increase $\Delta R_{2}$ tends to infinity. This wave length corresponds to the critical wave length, that is, $\Omega=\omega_{0} U / g=1 / 4$. For $\lambda<15 \mathrm{~m}$, the diffracted wave does not exist in front of the foil.

For the critical value and small values of $\lambda$, $\Delta R_{2}$ becomes larger than $\bar{T}$, and $\bar{T}_{a}$ becomes negative. Hence, for these values of $\lambda$, the linear Wells turbine can not produce thrust.

In Fig. 6, the lift $L$ and the moment $M$ acting on the same linear Wells turbine as in Fig. 5 are shown, where $\operatorname{Amp}()$ and $\operatorname{Arg}($ ) refer to the amplitude and the argument of the quantity in the parentheses respectively. When $\lambda$ tends to infinity, the reduced frequency tends to zero, and $\operatorname{Arg}(L)$ and $\operatorname{Arg}(M)$ tends to $90 \mathrm{deg}$.

In Fig. 7, the performance of a linear Wells turbine of chord $2 l=0.328 \mathrm{~m}$ advancing with various speeds in a wave of wave length $\lambda=8 \mathrm{~m}$ is shown. In this case, $U=0.73 \mathrm{~m} / \mathrm{s}$ is the critical speed where $\Omega=1 / 4$.

It must be noted that $\bar{T}_{a}$ becomes negative for $U>2.47 \mathrm{~m} / \mathrm{s}$. The existence of the speed limit can be found only by introducing the free surface effect and is not mentioned in Wu's papes ${ }^{15), 16)}$.

In Figs. 8 and 9, results for following seas are shown. In following seas, $\Delta R_{2}$ is always negative 

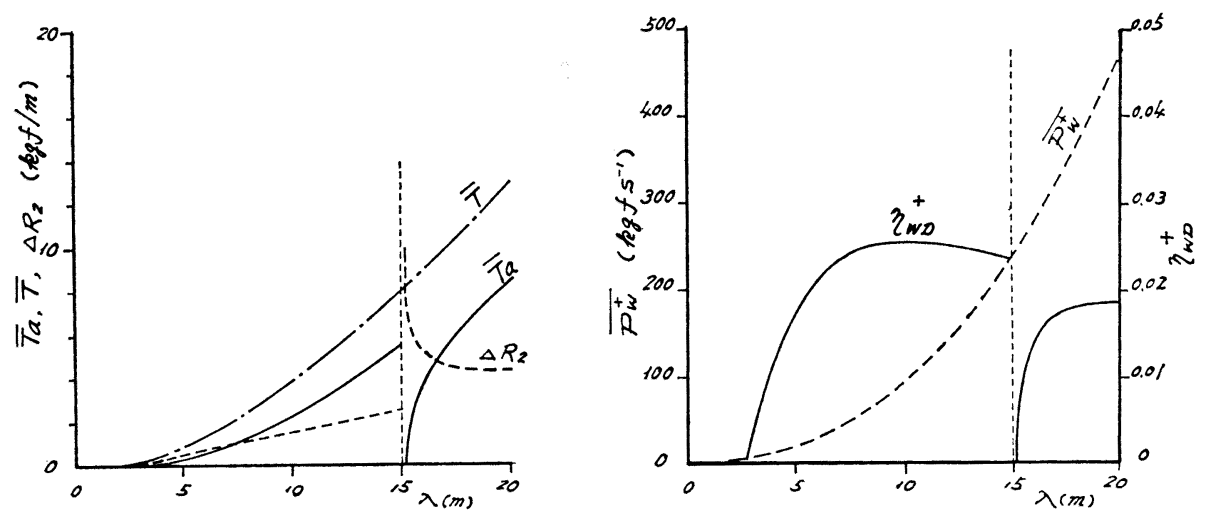

Fig. 5 Performance of a linear Wells turbine in head seas. $\left(U=1 \mathrm{~m} / \mathrm{s}, h_{1}=0.492 \mathrm{~m}, l=0.164 \mathrm{~m}, a=\lambda / 40\right)$
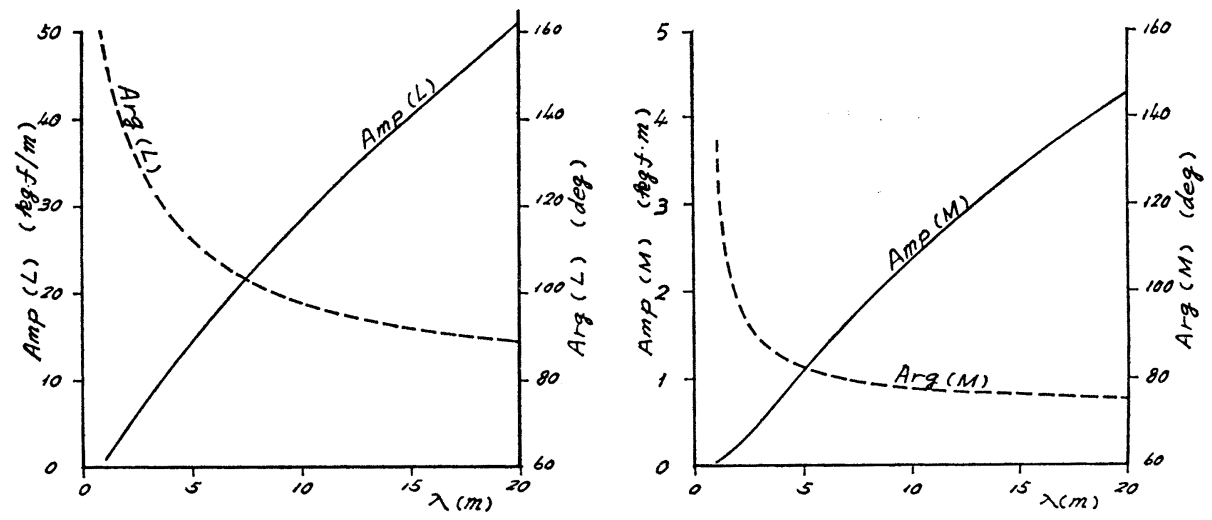

Fig. 6 Lift $L$ and moment $M$ acting on a linear Wells turbine in head seas. $\left(U=1 \mathrm{~m} / \mathrm{s}, \quad h_{1}=0.492 \mathrm{~m}, l=0.164 \mathrm{~m}, a=\lambda / 40\right)$
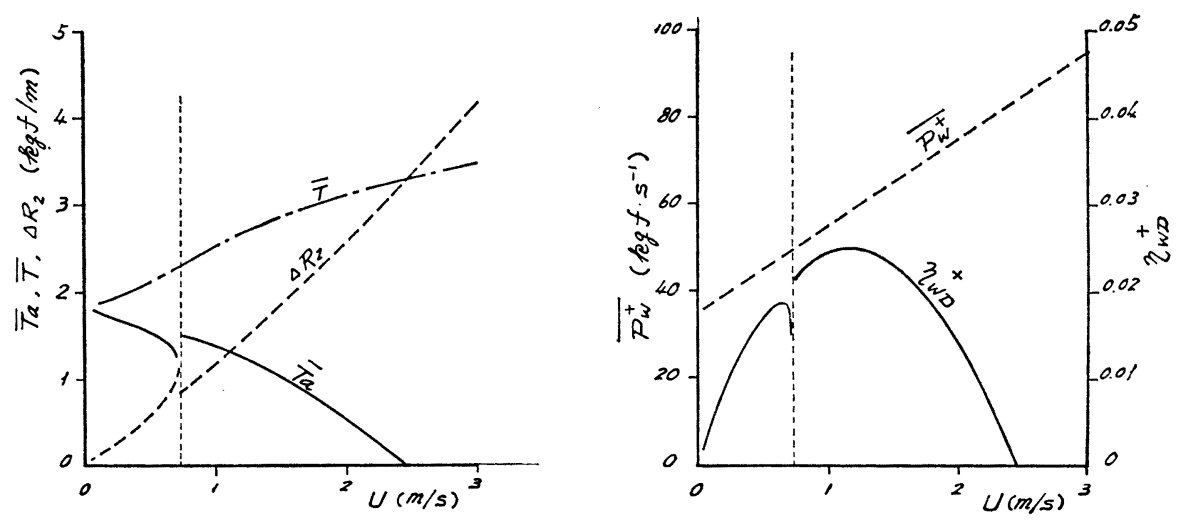

Fig. 7 Performance of a linear Wells turbine in a head sea. $\left(\lambda=8 \mathrm{~m}, h_{1}=0.492 \mathrm{~m}, l=0.164 \mathrm{~m}, a=0.2 \mathrm{~m}\right)$ 

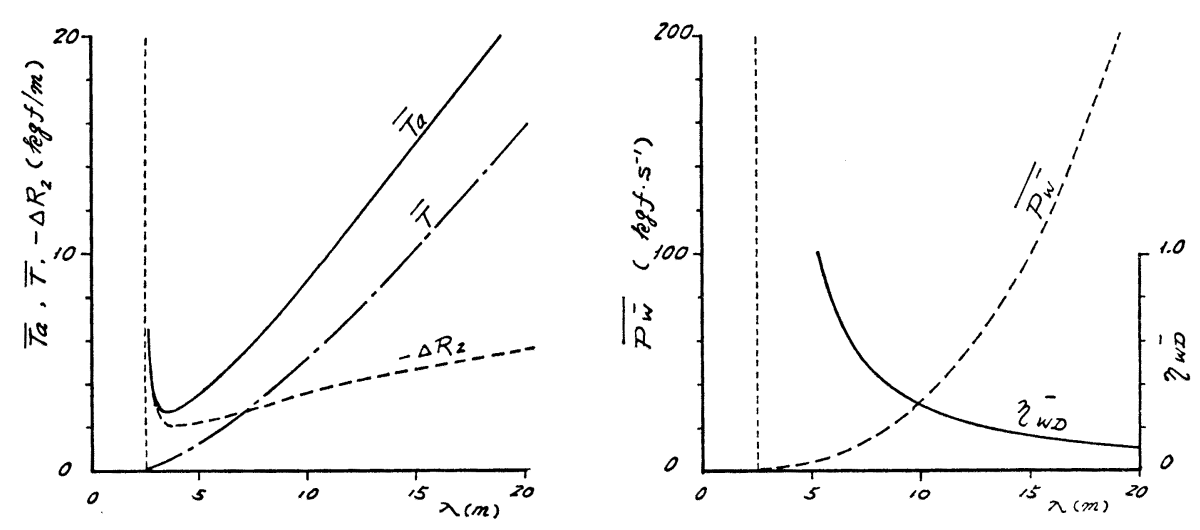

Fig. 8 Performance of a linear Wells turbine in following seas. $\left(U=1 \mathrm{~m} / \mathrm{s}, h_{1}=0.492 \mathrm{~m}, l=0.164 \mathrm{~m}, a=\lambda / 40\right)$
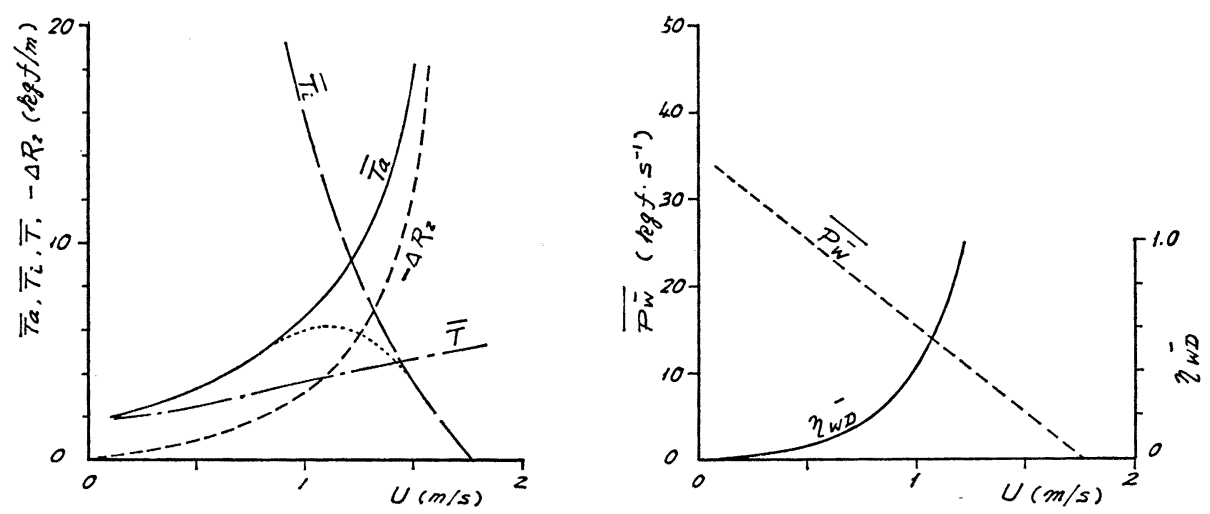

Fig. 9 Performance of a linear Wells turbine in a following sea. $\left(\lambda=8 \mathrm{~m}, h_{1}=0.492 \mathrm{~m}, l=0.164 \mathrm{~m}, a=0.2 \mathrm{~m}\right)$

and makes $\bar{T}_{a}$ larger than $\bar{T}$ calculated by Wu's theory. Hence, applications of linear Wells turbine in following seas may have some practicability.

In Fig. $8, \lambda=2.5 \mathrm{~m}$ is the critical wave length. Hence, for $\lambda<2.5 \mathrm{~m}$, the group velocity of the wave $c_{g}$ becomes smaller than the advance speed of the foil, and the foil can not produce thrust by absorbing wave power.

In Fig. 9, $U=1.76 \mathrm{~m} / \mathrm{s}$ is the critical speed, and, for $U>1.76 \mathrm{~m} / \mathrm{s}, \quad c_{g}$ of the wave of $\lambda=8 \mathrm{~m}$ becomes smaller than $U$. Hence, the foil can not produce thrust. But when $U$ tends to $1.76-0 \mathrm{~m} / \mathrm{s}$, $\Delta R_{2}$ tends to infinity. This is clearly the error due to the approximate procedure adapted in the present paper to estimate the resistance increase.

The ideal thrust $\bar{T}_{i}$ :

$$
\bar{T}_{i}=\tilde{P}_{W}^{-} / U
$$

is shown in Fig. 9. If a more refined theory is used to estimate $\bar{T}_{a}, \bar{T}_{a}$ may tends to zero when $U$ tends to the critical value as shown by a dotted line in Fig. 9.

\section{Conclusions}

In the present paper, the following items are discussed.

(1) The possibility of the wave devouring propulsion in head seas is shown from a general viewpoint of conversion of wave power into thrust.

(2) Wu's theory ${ }^{15), 16)}$ for oscillating foil propulsors in waves is developed by including approximately the free surface effect. For the purpose, the two-dimensional version of Maruo's theory for resistance increase in waves $^{9)}$ is applied.

(3) Some numerical results are given for nonoscillating foils in waves or linear Wells turbines are given, and the importance of introducing the free surface effect is shown. 


\section{Acknowledgements}

The author is deeply indebted to Dr. Y. Terao of Tokai University for his making various types of wave devouring propulsion devices and showing the author the experiments. Experiments by Dr. Terao and the discussions with him were very helpful for the present research.

The author acknowledges favours given to him by Dr. S. Matsuki of Kobe University of Mercantile Marine and Prof. S. Naito of Osaka University, since they informed the author the existence of refs. 11) and 3) respectively. The discussions with Prof. Naito about the wave drifting force were very useful to the author.

Mr. M. Murakami of Hitachi Shipbuilding \& Engineering Co., Ltd. is also sincerely acknowledged for his big help given to the author in the preparation of the manuscript of the present paper.

\section{References}

1) Bessho, M. \& Kyozuka, Y.: Propulsion by Making the Waves (Preliminary Observation of Its Feasibility), JTTC I (No. 52), Soc. of Naval Architects of Japan, 1980-1-06 (52-3-2), (1980/June) in Japanese.

2) Hanaoka, T.: Non-Uniform Theory of Wave Resistance (Part 1-Two Dimensional Fluid Field), J. of Zosen Kiokai, No. 89 (1956), in Japanese.

3) Higo, Y., Nakamura, S. \& Takagi, M.: A Study on the Resistance Increase of a Towed Pontoon Among the Waves, J. of Kansai Soc. of Naval Architects, No. 174 (1979), pp. 45 56, in Japanese.

4) Huse, E.: Wave Induced Mean Force on Platforms in Direction Opposite to Wave Propagation, Norwegian Maritime Res., No. 1 (1977), pp. 2 5.

5) Isshiki, H.: Thrust Increase by Wave Absorption, JTTC II (No. 64), Soc. of Naval Architects of Japan, 1981-II-05 (SK 64-9), (1981/June), in Japanese.

6) Lighthill, M. J.: Aquatic Animal Propulsion of High Hydrodynamical Efficiency,
J. Fluid Mech., Vol. 44, part 2 (1970), pp. $265 \sim 301$.

7) Longuet-Higgins, M. S.: The Mean Forces Exerted by Waves on Floating or Submerged Bodies with Applications to Sand Bars and Wave Power Machines, Proc. R. Soc. Lond. A. 352 (1977), pp. 463 480.

8) Maruo, H.: The Drift of a Body Floating on Waves, J. Ship Res. (1960/Dec.), pp. $1 \sim 10$.

9) Maruo, H.: Resistance in Waves, chap. 5, of 60TH ANNIVERSARY SERIES, Vol. 8, The Soc. of Naval Architects of Japan (1963), pp. 67 102 .

10) Newman, J. N.: MARINE HYDRODYNAMICS, The MIT Press (1977), p. 228.

11) Schult, J.: CURIOUS BOATING INVENTIONS, translated by I. Moore, Paul Elek, London, p. 42.

12) Terao, Y.: A Floating Structure Which Moves Towards the Waves (Possibility of Wave Devouring Propulsion), J. of Kansai Soc. of Naval Architects, No. 184 (1982), in Japanese.

13) Wu, T. Y.: Hydrodynamics of Swimming Propulsion. Part 1. Swimming of a TwoDimensional Flexible Plate at Variable Forward Speeds in an Inviscid Fluid, J. Fluid Mech., Vol. 46, part 2 (1971), pp. $337 \sim 355$.

14) Wu, T. Y.: Hydrodynamics of Swimming Propulsion. Part 2. Some Optimum Shape Problems, J. Fluid Mech., Vol. 46, part 3 (1971), p. 521 544.

15) Wu, T. Y.: Extraction of Flow Energy by a Wing Oscillating in Waves, J. Ship Res. (1972/Mar.), pp. 66 78.

16) Wu, T. Y. \& Chwang, A. T.: Extraction of Flow Energy by Fish and Birds in a Wavy Stream, SWIMMING AND FLYING IN NATURE, Vol. II edited by T. Y. Wu, C. J. Brokaw \& C. Brennan, Plenum Press, pp. $687 \sim 702$.

17) Wave Energy for Propelling Craft-Nothing New, The Naval Architect (1973/ Nov.), p. 239. 